Marquette University

e-Publications@Marquette

Mathematics, Statistics and Computer Science

Mathematics, Statistics and Computer Science,

Faculty Research and Publications

Department of

$11-1-2013$

\title{
Progressive Reliability Method and Its Application to Offshore Mooring Systems
}

Mir Emad Mousavi

Texas A \& M University

Paolo Gardoni

University of Illinois at Urbana-Champaign

Mehdi Maadooliat

Marquette University, mehdi.maadooliat@marquette.edu

Accepted version. Engineering Structures, Vol. 56 (November 2013): 2131-2138. DOI. (C) 2013

Elsevier. Used with permission. 


\title{
Progressive Reliability Method and Its Application to Offshore Mooring Systems ${ }^{1}$
}

\author{
Mir Emad Mousavi ${ }^{1}$, Texas A\&M University, TAMU - 3136, College Station, TX 77043 \\ 1 Present Address: Aker Solutions Inc., 3010 Briarpark Dr. Suite 500, Houston, TX 77042, emad.mousavi@akersolutions.com, 713-981-12047
}

(corresponding author)

Paolo Gardoni, Department of Civil and Environmental Engineering, University of Illinois at UrbanaChampaign

Mehdi Maadooliat, Department of Mathematics, Statistics and Computer Science, Marquette University, Milwaukee, Wisconsin, USA

\begin{abstract}
Assessing the reliability of complex systems (e.g. structures) is essential for a reliability-based optimal design that balances safety and costs of such systems. This paper proposes the Progressive Reliability Method (PRM) for the quantification of the reliability of complex systems. The proposed method is a closed-form solution for calculating the probability of failure. The new method is flexible to the definition of "failure" (i.e., can consider serviceability and ultimate-strength failures) and uses the rules of probability theory to estimate the failure probability of the system or its components. The method is first discussed in general and then illustrated in two examples, including a case study to find the safest configuration and orientation of a 12-line offshore mooring system. The PRM results are compared with results of a similar assessment based on the Monte Carlo simulations. It is shown in the example of twocomponent that using PRM, the importance of system components to system safety can be quantified and compared as input information for maintenance planning.
\end{abstract}

\section{Introduction}

The safety and cost-effectiveness of a system can be maximized if a reliability-based method is used for its design. For example, the safety of a structural system usually depends on numerous random variables, including environmental demands (loads), material properties and structural capacities (strengths.) Therefore, the safety of such system is uncertain. In practice, for the design of reliable structures, a number of safety criteria are fulfilled as recommended by design codes, normally through the use of factors of safety (FoS). Using FoSs that locally assesses the system safety has been practiced for many years and has produced structures that function under various circumstances. However, concerns remain in terms of the system reliability. Using FoS provides little quantitative information about the overall reliability of the structural systems. In turn, quantitative reliability methods quantify the system safety and reliability in terms of their failure probability and provide a basis for changing and optimizing the design variables to achieve sufficiently safe structures that are not overdesigned.

This paper proposes a Progressive Reliability Method (PRM) as a closed-form solution for quantifying the system reliability using the basic rules of the probability theory. This general method can compute the probability of system failure in terms of its functionality and performance or the failure of certain system components (this method is flexible to the definition of system failure.) For example, in structural

\footnotetext{
${ }^{1}$ Mousavi, Mir Emad, Paolo Gardoni, and Mehdi Maadooliat. "Progressive reliability method and its application to offshore mooring systems." Engineering Structures 56 (2013): 2131-2138.
} 
engineering, the maximum deformation of the structure to a certain level or the ultimate-strength failure of one or some of the system components usually defines the system failure. In a PRM, the reliability assessment accounts for the possible progressive failures of the system components until the system failure criterion is met. Such assessment requires calculating the probability of various failure scenarios and calculating the probability of system failure under each scenario. Because after each component failure, the probability space of the remaining components changes (due to the redistribution of the demands), and any damaged state of the system may occur through multiple failure scenarios, a general solution that accounts for all such complexities is developed.

The PRM uses known rules of probability to formulate a general method for calculating the reliability of complex systems. The proposed method provides a closed-form solution that accounts for the dynamic changes in the stochastic model associated with the possible progressive failures of the components. This general method captures the complexities that are not captured when only one or two failures of a limited number of components are considered. For example, multiple paths can lead to the same damaged systems with several failed components. By using the well-known total probability rule, converting the problem to a fragility (conditional probability of system failure given system demands) problem, and making the assumption that only one component fails at a time, PRM is a novel formulation based on known concepts to compute the probability of failure of complex systems.

A PRM can be applied in the design of many complex systems. As an illustration, this paper shows how the proposed method can be applied to an offshore mooring system. Offshore mooring systems are important structures, especially in development of oil and gas drilling and production systems. The main function of a mooring system is to maintain a floating structure within a certain offset of its still position. The offshore industry has used several mooring systems in deepwaters; however, some of them have failed during service or extreme conditions. A mooring system consists of multiple mooring lines. Each line includes a few components. Because of the uncertainties in the strength of the system components and the environmental loads, a reliability-based design optimization can improve the design of the mooring systems more accurately than the conventional FoS-based methods. Furthermore, the failure of any individual component in a mooring system does not necessarily compromise its functionality; therefore, a PRM can be used for an unbiased and comprehensive reliability-based optimization.

Calculating the probability of failure of offshore mooring systems or their components has been the subject of a number of studies in the last few years. Stiff et al. [1] calculated the probability of failure of mooring systems using a closed-form solution accounting for the ultimate-strength (overloading) failure only and based on a quadratic model for the failure of the individual line components. Shah et al. [2] assessed the reliability of mooring systems by calculating the probability that incoming waves have a frequency near the natural frequency of the mooring system. The reliability of individual components and individual lines in a mooring system was discussed by Vazquez-Hernandez et al. [3] and MontesIturrizaga et al. [4] concerning the design criteria and a method to quantify the probability of a component failure in an intact system. Grimea and Langley [5] used a simplified model to compare the probability of failure of mooring systems under different extreme environmental loads. Ku et al. [6] performed a limited number of Monte Carlo simulations to estimate the probability of mooring system failure with various system properties. The probability of failure of suction caissons in mooring systems was discussed by Najjar and Gilbert [7]. Montes-Iturrizaga et al. [8] also analyzed individual mooring lines for their reliability and used a new equation to calculate the probability of failure of the individual lines in the 
intact system. Mousavi and Gardoni [9] calculated the probability of failure of mooring systems using Monte Carlo simulation and a new definition for the failure based on system functionality by making the assumption that one mooring component fails at a time.

The main function of a mooring system is to maintain a vessel within an allowable offset of its still position. However, a review of the existing studies on the calculation of the probability of failure of mooring systems shows that the probability of serviceability and functionality failure (the probability that the offset of a moored vessel exceeds an allowable limit) is typically not considered. Mousavi and Gardoni [9] discussed this probability, suggesting a method to evaluate the probability of serviceability failure in an offshore mooring system. However, the proposed method is based on the Monte Carlo simulations. The simulation-based methods are used for the reliability analysis of other complex systems considering serviceability limit states. For example, Hernandez-Fajardo (Hernandez-Fajardo 2012) used a Monte Carlo analysis to quantify the reliability of urban systems under seismic hazard accounting for the interdependency of the component fragilities. However, the simulation-based methods are usually time consuming and require extensive analyses. The proposed PRM can quantify the reliability significantly faster than such simulation-based approaches, accounting for the dependency of the component failures using the total probability rule and by computing the conditional probabilities of failure of components under all relevant configurations. PRM can also be used to quantify the likelihood of each scenario of system failure and compare the importance of the components to maintain system safety. A simplified example is used in the paper to highlight such application.

In the following sections, the PRM is first formulated in general terms and explained also using an idealized system with two components. Then, as an illustration, it is used in two case studies for mooring systems.

\section{Progressive Reliability Method (PRM)}

A PRM calculates the failure probability of a structural system accounting for the progressive failure of system components until the system fails. In this method, two important terminologies are essential and frequently referred to, which are a) a configuration and b) a failure scenario. We will first introduce these terms and then discuss the method.

\section{System Configurations and Failure Scenarios}

We define a configuration as any possible state of the structure in terms of the possible state (failure or no failure) of its components. This definition is based on the assumption that any component may fail and is always either in the state of failed or in the state of not failed. Any structural system has an initial arrangement of its components. We call the initial state of a structural system (with no failed components) as the intact configuration. Under the effect of environmental loads, some components may fail. Any component failure leads the system to a new configuration. If a system has $N$ components that may potentially fail, the total number of possible configurations is $2^{N}$, which includes the intact and $2^{N}-1$ unique damaged configurations (including a configuration with all components failed). A system configuration can be shown using a binary row vector with $N$ columns so that each column refers to the status of a component under that configuration; if component $j$ fails, the $j^{\text {th }}$ column is 1 ; otherwise it is 0 . We refer to each configuration with $\mathbf{X}_{i}$, where the index $i$ is the decimal representation of the binary 
vector corresponding to that configuration. The intact and the most damaged (all components failed) configurations are therefore shown as $\mathbf{X}_{0}=\underbrace{[000 \ldots 0]}_{N}$ and $\mathbf{X}_{2^{N}-1}=\underbrace{[111 \ldots 1]}_{N}$, respectively.

We define a failure scenario of $\mathbf{X}_{i}$ as a group of steps (or the path) that leads the system from $\mathbf{X}_{0}$ (the intact configuration) to $\mathbf{X}_{i}$. In general, the occurrence of $\mathbf{X}_{i}$ can be through one or more failure scenarios. However, because in a structural system, the environmental loads usually vary within a time frame until reaching to a maximum load, and that the capacity of the components are random, it is almost impossible that in more than one component, the internal force exceed the capacity at the same time. Therefore, as suggested by Mousavi and Gardoni [9], we assume that one component fails at a time. This assumption means that after each component failure, any next failure may occur only after the loads are redistributed among the remaining components under the new configuration. With this assumption, if $\mathbf{X}_{i}$ has $n_{i}$ failed components, the total number of possible unique failure scenarios leading to this configuration are:

$$
K_{i}=n_{i} !
$$

Eq. (1) shows that if $\mathbf{X}_{i}$ has more than one failed components, multiple failure scenario is possible. We show the $k^{\text {th }}$ possible failure scenario of $\mathbf{X}_{i}$ by $\tilde{\mathbf{X}}_{i}^{(k)} . \tilde{\mathbf{X}}_{i}^{(k)}$ is a matrix in which the first row is a binary vector describing the intact system, $\mathbf{X}_{0}$, the middle rows (from the $2^{\text {nd }}$ to the $n_{i}^{\text {th }}$ row) are binary vectors describing the configurations that occur in between, and the last row is $\mathbf{X}_{i}$. The number of rows in this matrix is $n_{i}+1$, since it includes a row for the intact system and $n_{i}$ failure steps. We show the $r^{\text {th }}$ row of the $k^{\text {th }}$ possible failure scenario as $\tilde{\mathbf{X}}_{i}^{(k, r)}$, and we denote the $j^{\text {th }}$ column of $\tilde{\mathbf{X}}_{i}^{(k, r)}$ as $\tilde{x}_{i}^{(k, r, j)}$, which is the status of component $j$ in the $r^{\text {th }}$ step of this failure scenario.

The notation used for the configurations and failure scenarios can be illustrated in an example. In a system with 8 components, we show the configuration that the first and the third components failed

using $\mathbf{X}_{5}=[00000101]$, where the index 5 is the decimal number equivalent to the binary vector in the brackets. As suggested by Eq.(1), $\mathbf{X}_{5}$ has two failure scenarios. They are:

$$
\tilde{\mathbf{X}}_{5}^{(1)}=\left[\begin{array}{l}
00000000 \\
00000001 \\
00000101
\end{array}\right], \tilde{\mathbf{X}}_{5}^{(2)}=\left[\begin{array}{l}
00000000 \\
00000100 \\
00000101
\end{array}\right]
$$

We also note that the intact configuration of a system always has only one scenario, which is $\tilde{\mathbf{X}}_{0}^{(1)}=[00000000]$ in this example.

\section{Fragility Model}

In a PRM, the conditional probability of system failure under a given environmental load depends on the probability that each configuration occurs as well as the probability that the system fails under that configuration and demand. The configurations of a system constitute a finite set of mutually exclusive and 
collectively exhaustive events because they are all unique and span all the possible options. Therefore, for a given demand (environmental load) $d_{E}$, we can calculate the conditional probability of failure, or fragility $[11,12]$, as

$$
\mathrm{P}\left(g_{s} \leq 0 \mid d_{E}\right)=\sum_{i=0}^{2^{N}-1}\left\{\mathrm{P}\left(g_{s} \leq 0 \mid \mathbf{X}_{i}, d_{E}\right) \times \mathrm{P}\left(\mathbf{X}_{i} \mid d_{E}\right)\right\}
$$

where $g_{s}$ is the system limit-state function defined such that $\mathrm{P}\left(g_{s} \leq 0 \mid \mathbf{X}_{i}, d_{E}\right)$ indicates the probability of system failure under $\mathbf{X}_{i}$ and $d_{E}$, and $\mathrm{P}(\cdot)$ denotes the probability of the event described in the parenthesis. Eq. (2) formulates the fragility of a structural system based on the PRM. By using the likelihoods of the demands, the overall system failure probability can then be calculated using the total probability rule [13]

$$
\begin{aligned}
\mathrm{P}\left(g_{s} \leq 0\right) & =\int_{B} \mathrm{P}\left(g_{s} \leq 0 \mid d_{E}\right) \times f\left(d_{E}\right) \mathrm{d} d_{E}=\int_{B}^{2^{N}-1} \sum_{i=0}^{1}\left\{\mathrm{P}\left(g_{s} \leq 0 \mid \mathbf{X}_{i}, d_{E}\right) \times \mathrm{P}\left(\mathbf{X}_{i} \mid d_{E}\right)\right\} \times f\left(d_{E}\right) \mathrm{d} d_{E} \\
& =1-\int_{B}^{2^{N}-1} \sum_{i=0}\left\{\mathrm{P}\left(g_{s}>0 \mid \mathbf{X}_{i}, d_{E}\right) \times \mathrm{P}\left(\mathbf{X}_{i} \mid d_{E}\right)\right\} \times f\left(d_{E}\right) \mathrm{d} d_{E}
\end{aligned}
$$

where $f(\cdot)$ is the probability density function (PDF) and $B$ is the domain of $d_{E}$.

Eqs. (2) and (3) indicate that the number of possible configurations in a system exponentially increases by increasing the number of components, implying that numerous deterministic analyses should be completed to evaluate the terms in these equations. However, as will be discussed later, it is expected that the term $\mathrm{P}\left(g_{s}>0 \mid \mathbf{X}_{i}, d_{E}\right)$ becomes 0 or negligible for most configurations with multiple failed components. For any such $\mathbf{X}_{i}$ and $d_{E}$, the rest of the terms in Eq. (3) become irrelevant. Moreover, any larger demand on that configuration or any progressive failures following that configuration also become irrelevant (i.e., their conditional probabilities of system failure are 0 too.) Finally, if some of the components form groups of series subsystems so that the failure of any component in the group makes other components of that group irrelevant to the system safety, then one deterministic analysis is sufficient to account for the failure of each series subsystem under a given demand and configuration. Therefore, to use Eq. (3), we only need to analyze the system for a limited number of configurations and range of demands with nonzero $\mathrm{P}\left(g_{s}>0 \mid \mathbf{X}_{i}, d_{E}\right)$. Hence, the decay of $\mathrm{P}\left(g_{s}>0 \mid \mathbf{X}_{i}, d_{E}\right)$ with progressive failures has an important role on reducing the analyses of the PRM, because it identifies the configurations and the range of demands that are irrelevant in Eqs. (3).

\section{Calculating the Conditional Probability that System Does Not Fail under Each Configuration}

Eq. (3) requires calculating $\mathrm{P}\left(g_{s}>0 \mid \mathbf{X}_{i}, d_{E}\right)$ for all the relevant $\mathbf{X}_{i}$ and $d_{E}$. This conditional probability depends on the definition of system failure. For example, a structural failure can be defined based on the ultimate-strength failure of some structural components, the serviceability of the structure, or a combination of these two. If a system failure is defined based on the ultimate-strength failure of certain components or certain number of components in a system, then if in $\mathbf{X}_{i}$, those components or number of components are already failed, then $\mathrm{P}\left(g_{s}>0 \mid \mathbf{X}_{i}, d_{E}\right)$ is 0 ; otherwise, it is 1 . Similarly, if a system 
failure is defined by a certain limit for the system response (e.g. deformation in a structure) and the modeling error is negligible, then $\mathrm{P}\left(g_{s}>0 \mid \mathbf{X}_{i}, d_{E}\right)$ is either 0 (when the failure criterion is met) or 1 (when the failure criterion is not met). However, if the modeling error is not negligible, then $\mathrm{P}\left(g_{s}>0 \mid \mathbf{X}_{i}, d_{E}\right)$ is a number between 0 and 1 that should be estimated using a reliability analysis (e.g. FORM, SORM).

\section{Calculating the Conditional Probability of Each Configuration}

According to Eq. (3), for calculating the system failure probability, we should calculate the conditional probability of each configuration given the demand on the system. Because any configuration $\mathbf{X}_{i}$ can occur through any of the $K_{i}$ failure scenarios leading to this configuration, we need to determine the conditional probabilities of all these failure scenarios to compute the conditional probability of this configuration. The failure scenarios of each configuration are mutually exclusive and collectively exhaustive; hence, we have

$$
\mathrm{P}\left(\mathbf{X}_{i} \mid d_{E}\right)=\mathrm{P}\left(\bigcup_{k=1}^{K_{i}} \tilde{\mathbf{X}}_{i}^{(k)} \mid d_{E}\right)=\sum_{k=1}^{K_{i}} \mathrm{P}\left(\tilde{\mathbf{X}}_{i}^{(k)} \mid d_{E}\right)
$$

where

$$
\mathrm{P}\left(\tilde{\mathbf{X}}_{i}^{(k)} \mid d_{E}\right)=\mathrm{P}\left(\bigcap_{\left\{j: \tilde{x}_{i}^{\left(k, m_{i}+1, j\right)}=0\right\}} g^{j}>0 \mid \mathbf{X}_{i}, d_{E}\right) \times \mathrm{P}\left(\bigcap_{r=1}^{n_{i}+1} \tilde{\mathbf{X}}_{i}^{(k, r)} \mid d_{E}\right)
$$

in which $g^{j}$ is the limit-state function of component $j$ and is non-positive when this component fails:

$$
g^{j}=c^{j}-d^{j}
$$

where $c^{j}$ and $d^{j}$ are the capacity and the demand of component $j$, respectively. Eq. (6) implies that if we know the probability density function (PDF) of $c^{j}$ and calculate $d^{j}$ given $\mathbf{X}_{i}$ and $d_{E}$, we can calculate the fragility of this component $[6,7,8]$. In Eq. (5), the first term calculates the conditional probability of no failure in the remaining components given the probability space of $\mathbf{X}_{i}$ and $d_{E}$, and the second term computes the conditional probability of previous failure steps that lead to this probability space given $d_{E}$. Assuming that given $\mathbf{X}_{i}$ and $d_{E}$, the failures of the components are independent from each other, for the first term of Eq. (5) we have

$$
\mathrm{P}\left(\bigcap_{\left\{j: x_{i}^{\left(k, m_{i}+1, j\right)}=0\right\}} g^{j}>0 \mid \mathbf{X}_{i}, d_{E}\right)=\prod_{\left\{j: \tilde{x}_{i}^{\left(k, n_{i}+1, j\right)}=0\right\}}\left\{1-\mathrm{P}\left(g^{j} \leq 0 \mid \mathbf{X}_{i}, d_{E}\right)\right\}
$$

Also, for the second term of Eq. (5), considering the Markov property of the steps of $\tilde{\mathbf{X}}_{i}^{(k)}$ [14] (which is that any further failure in the system given its current state is independent from the previous failure steps that led to this state), for any $\rho$ between 1 and $n_{i}$, we can write 
$\mathrm{P}\left(\bigcap_{r=1}^{\rho+1} \tilde{\mathbf{X}}_{i}^{(k, r)} \mid d_{E}\right)=\mathrm{P}\left(\tilde{\mathbf{X}}_{i}^{(k, \rho+1)} \mid \tilde{\mathbf{X}}_{i}^{(k, \rho)}, d_{E}\right) \times \mathrm{P}\left(\bigcap_{r=1}^{\rho} \tilde{\mathbf{X}}_{i}^{(k, r)} \mid d_{E}\right)$

where

$$
\mathrm{P}\left(\tilde{\mathbf{X}}_{i}^{(k, \rho+1)} \mid \tilde{\mathbf{X}}_{i}^{(k, \rho)}, d_{E}\right)=\zeta_{i}^{(k, \rho)} \times\left\{\mathrm{P}\left(g^{\tilde{j}} \leq 0 \mid \tilde{\mathbf{X}}_{i}^{(k, \rho)}, d_{E}\right) \times \prod_{\left\{j: \tilde{x}_{i}^{(k, \rho, j)}=0 \wedge j \neq \tilde{j}\right\}}\left[1-\mathrm{P}\left(g^{j} \leq 0 \mid \tilde{\mathbf{X}}_{i}^{(k, \rho)}, d_{E}\right)\right]\right\}
$$

Here $\tilde{j}$ indicates the component that is failing in the latest step (only $\tilde{j}$ satisfies $\tilde{x}_{i}^{(k, \rho+1, \tilde{j})}-\tilde{x}_{i}^{(k, \rho, \tilde{j})}=1$.) Eq. (8) recursively computes the conditional probability of the first $\rho$ failure steps of $\tilde{\mathbf{X}}_{i}^{(k)}$ given the initial condition of $\mathrm{P}\left(\tilde{\mathbf{X}}_{i}^{(k, 1)} \mid d_{E}\right)=1$ for any $k, d_{E}$, and $i$ (because the system is always initially in the intact configuration), and Eq. (9) determines the conditional probability of each failure step of $\tilde{\mathbf{X}}_{i}^{(k)}$ given that the previous failure step already has occurred. In Eq. (9), $\zeta_{i}^{(k, \rho)}$ is an adjustment factor that takes into account the one-at-a-time-failure assumption. This adjustment is necessary because, given $\tilde{\mathbf{X}}_{i}^{(k, \rho)}$ and $d_{E}$, the summation of the conditional probabilities of all the possible immediate events (failure or no failure) after each failure step must be 1 . Hence

$$
\zeta_{i}^{(k, \rho)}=\frac{\left[1-\prod_{\left\{j: \tilde{x}_{i}^{(k, \rho, j)}=0\right\}}\left\{1-\mathrm{P}\left(g^{j} \leq 0 \mid \tilde{\mathbf{X}}_{i}^{(k, \rho)}, d_{E}\right)\right\}\right]}{\sum_{\left\{\tilde{j}: \tilde{x}_{i}^{(k, \rho, j)}=0\right\}}\left[\mathrm{P}\left(g^{\tilde{j}} \leq 0 \mid \tilde{\mathbf{X}}_{i}^{(k, \rho)}, d_{E}\right) \prod_{\left\{j: \tilde{x}_{i}^{(k, \rho, j)}=0 \wedge j \neq \tilde{j}\right\}}\left\{1-\mathrm{P}\left(g^{j} \leq 0 \mid \tilde{\mathbf{X}}_{i}^{(k, \rho)}, d_{E}\right)\right\}\right]}
$$

In Eq. (10), the numerator is the conditional probability of any further failure given $\tilde{\mathbf{X}}_{i}^{(k, \rho)}$ and $d_{E}$, and the denominator is the summation of the conditional probabilities of all the possible immediate one-at-atime-failure events given $\tilde{\mathbf{X}}_{i}^{(k, \rho)}$ and $d_{E}$.

We can use Eqs. (5-10) to compute the conditional probability of each $\tilde{\mathbf{X}}_{i}^{(k)}$ and then Eq. (4) to calculate the conditional probability of $\mathbf{X}_{i}$ given $d_{E}$.

\section{Deterministic Analysis}

A PRM requires deterministic analysis of all the system configurations $\left(\mathbf{X}_{i}\right)$ and demands $\left(d_{E}\right)$ with $\mathrm{P}\left(g_{s}>0 \mid \mathbf{X}_{i}, d_{E}\right)>0$. To do so, we should first discretize the environmental loads and calculate the response of the intact configuration and find the range of $d_{E}$ within $B$ that $\mathrm{P}\left(g_{s}>0 \mid \mathbf{X}_{0}, d_{E}\right)>0$. This range is also the largest range of $d_{E}$ that should be included in deterministic analysis of all the other configurations because all other configurations are products of failure in the intact system. We call this 
range of $d_{E}$ as the functional range of demands. We should then consider $N$ new configurations where each of them has one fewer component compared to the intact configuration. For each new $\mathbf{X}_{i}$, deterministic analysis should be performed. Then, only if $\mathrm{P}\left(g_{s}>0 \mid \mathbf{X}_{i}, d_{E}\right)>0$ within the functional range of demands, all possible new configurations with one fewer components should be considered. The functional range of demands with $\mathrm{P}\left(g_{s}>0 \mid \mathbf{X}_{i}, d_{E}\right)>0$ may shrink for each new configuration and thus for other configurations with further failures. Analysis of configurations with progressive failure should continue until $\mathrm{P}\left(g_{s}>0 \mid \mathbf{X}_{i}, d_{E}\right)$ becomes 0 or negligible for any $d_{E}$, which determines an end to that path of progressive failures that must be considered. At the end of deterministic analysis, the internal forces for all the demands and configurations with $\mathrm{P}\left(g_{s}>0 \mid \mathbf{X}_{i}, d_{E}\right)>0$ are available and can be used to calculate the conditional probabilities of relevant configurations.

In summary, in a PRM analysis, after the deterministic analyses are completed for all the configurations and demands with $\mathrm{P}\left(g_{s}>0 \mid \mathbf{X}_{i}, d_{E}\right)>0$, all the corresponding failure scenarios have to be determined and their conditional probabilities must be computed. Then, using Eq. (4), the conditional probability of each configuration can be determined. Next, Eq. (2) gives the conditional probability of system failure. Finally, Eq. (3) computes the probability of failure of the system. The proposed formulation can be used also to compute the probability of exceeding a specified damage level by defining an appropriate system limit-state function $g_{s}$ so that $g_{s} \leq 0$ indicates exceedance of the selected damage level.

The computations for the PRM are illustrated using an example of a two-component structural system.

\section{Example of Two-component Structural System}

We illustrate the computations of the PRM using an example two-component structural system. In this example, given the environmental load, we calculate the conditional probabilities of 1) serviceability failure of the system, 2) ultimate-strength failure of the system (exceedance of one component failure in the system), and 3) failure of each component. The components of this system are two springs arranged in a parallel form. Each spring is under a tension of $d^{j}$ induced by $d_{E}$ and has a fixed stiffness of $k^{j}$ but an uncertain strength of $c^{j}$. The cylinder is a rigid element with negligible friction with the two sidewalls. Because of the side-walls, $d_{E}$ will be distributed among the active (not failed) component(s) as axial loads only. The capacity of each component is independent from the other. 


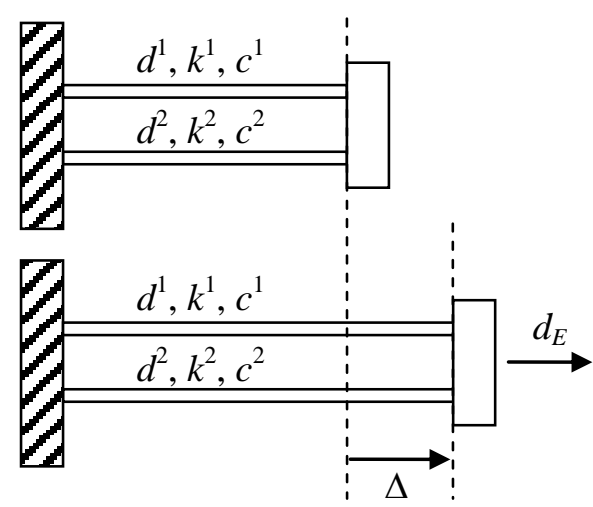

Figure 1 - Schematic view of the example two-component system

For this example, we define the serviceability failure as if $\Delta$, the cylinder displacement, exceeds $\Delta_{0}$, an allowable displacement,

$$
\mathrm{P}\left(g_{s}^{\text {serviceability }} \leq 0 \mid d_{E}\right)=\mathrm{P}\left(\Delta \geq \Delta_{0} \mid d_{E}\right)
$$

where, considering the basic physics of this system,

$$
\Delta=\frac{d_{E}}{\sum_{j=1}^{N=2}\left[k^{j} \times\left(1-x_{i}^{j}\right)\right]}
$$

in which $x_{i}^{j}$ indicates the status of each component and is 1 if component $j$ fails and is 0 otherwise, $(j$ is the component ID and is 1 or 2 in this example.) Eqs. (11) and (12) indicate that the conditional probabilities of serviceability failure cannot be calculated without conditioning on the statuses of the components because $\Delta$ is a function of $x_{i}^{j}$. Therefore, Eq. (2) can be used to compute this conditional probability as

$$
\mathrm{P}\left(g_{s}{ }^{\text {serviceability }} \leq 0 \mid d_{E}\right)=\sum_{i=0}^{2^{N}-1=3}\left\{\mathrm{P}\left(\Delta \geq \Delta_{0} \mid \mathbf{X}_{i}, d_{E}\right) \times \mathrm{P}\left(\mathbf{X}_{i} \mid d_{E}\right)\right\}
$$

We define the ultimate-strength failure of this system as if both the system components fail:

$$
\mathrm{P}\left(g_{s}^{\text {ultimate-strength }} \leq 0 \mid d_{E}\right)=\mathrm{P}\left(n=2 \mid d_{E}\right)
$$

where $n$ is the number of failed components in the system. This system has four possible configurations, which are:

$$
\mathbf{X}=\left\{\mathbf{X}_{0}, \mathbf{X}_{1}, \mathbf{X}_{2}, \mathbf{X}_{3}\right\}=\{[00],[01],[10],[11]\}
$$


Because the number of failed components is less than 2 for $\mathbf{X}_{0}, \mathbf{X}_{1}$, and $\mathbf{X}_{2}$ and is 2 for $\mathbf{X}_{3}$, based on Eq. (2), we can write

$\mathrm{P}\left(g_{s}{ }^{\text {ultimate-strength }} \leq 0 \mid d_{E}\right)=\sum_{i=0}^{3} \mathrm{P}\left(n_{i}=2 \mid \mathbf{X}_{i}, d_{E}\right) \times \mathrm{P}\left(\mathbf{X}_{i} \mid d_{E}\right)=\mathrm{P}\left(\mathbf{X}_{3} \mid d_{E}\right)$

The failure of each component is assessed using the component limit-state functions introduced in Eq. (6). In this example, we can write

$d^{j}=k^{j} \Delta \times\left(1-x_{i}^{j}\right)$

Thus, using Eq. (2), the conditional probability of failure of each component for this example will be

$$
\mathrm{P}\left(g^{j} \leq 0 \mid d_{E}\right)=\sum_{i=0}^{3}\left\{\mathrm{P}\left(g^{j} \leq 0 \mid \mathbf{X}_{i}, d_{E}\right) \times \mathrm{P}\left(\mathbf{X}_{i} \mid d_{E}\right)\right\}=\sum_{i=0}^{3}\left\{\mathrm{P}\left(c^{j} \leq d^{j} \mid \mathbf{X}_{i}, d_{E}\right) \times \mathrm{P}\left(\mathbf{X}_{i} \mid d_{E}\right)\right\}
$$

Eqs. (13), (16), and (18) calculate the system and component conditional probabilities of failure given $d_{E}$ and $\mathbf{X}_{i}$ based on the PRM.

Here, we calculate the conditional probabilities of system and component failures under $d_{E}$ for some arbitrary input information. We assume that the component capacity, $c^{j}$, follows a lognormal distribution. The component stiffness and the mean and standard deviation of each $c^{j}$ are given in Table 1. The environmental load $d_{E}$ and the allowable displacement $\Delta_{0}$ are taken to be $50 \mathrm{kN}$ and $1.2 \mathrm{~m}$, respectively.

Table 1. Properties of the Components in the Two-component Example System

\begin{tabular}{|c|c|c|c|}
\hline Component ID & $\mu_{c}{ }^{j}(\ln (\mathrm{m}))$ & $\sigma_{c}{ }^{j}(\ln (\mathrm{m}))$ & $k^{j}(\mathrm{kN} / \mathrm{m})$ \\
\hline 1 & 10 & 3 & $4.0 \times 10^{4}$ \\
\hline 2 & 15 & 4.5 & $4.0 \times 10^{5}$ \\
\hline
\end{tabular}

Considering the four possible configuration of this system in Eq. (15), five failure scenarios $\left\{\tilde{\mathbf{X}}_{0}^{(1)}, \tilde{\mathbf{X}}_{1}^{(1)}, \tilde{\mathbf{X}}_{2}^{(1)}, \tilde{\mathbf{X}}_{3}^{(1)}, \tilde{\mathbf{X}}_{3}^{(2)}\right\}$ are possible. Using the input information for this example and Eqs. (12) and (17), the system displacements, and the component demands and conditional probabilities of failure under $d_{E}$ and all the failure steps (and their corresponding configurations) are computed as shown in Table 2.

Table 2. Deterministic Results and Conditional probabilities of Component Failure under each $\tilde{\mathbf{X}}_{i}^{(k, \rho)}$

\begin{tabular}{|c|c|c|c|c|c|c|}
\hline $\begin{array}{c}\text { Failure } \\
\text { Step }\end{array}$ & $\begin{array}{c}\text { Corresponding } \\
\mathbf{X}_{i}\end{array}$ & $\Delta$ & $d^{1}$ & $d^{2}$ & $\mathrm{P}\left(g^{1} \leq 0 \mid \mathbf{X}_{i}, d_{E}\right)$ & $\mathrm{P}\left(g^{2} \leq 0 \mid \mathbf{X}_{i}, d_{E}\right)$ \\
\hline$\tilde{\mathbf{X}}_{i}^{(k, 1)}$ & $\mathbf{X}_{0}=[00]$ & 0.11 & $4.6 \times 10^{3}$ & $4.6 \times 10^{4}$ & 0.2994 & 0.1710 \\
\hline$\tilde{\mathbf{X}}_{1}^{(1,2)}=\tilde{\mathbf{X}}_{3}^{(1,2)}$ & $\mathbf{X}_{1}=[01]$ & 1.25 & $5.0 \times 10^{4}$ & - & 0.6077 & 1.0000 \\
\hline$\tilde{\mathbf{X}}_{2}^{(1,2)}=\tilde{\mathbf{X}}_{3}^{(2,2)}$ & $\mathbf{X}_{2}=[10]$ & 0.13 & - & $5.0 \times 10^{4}$ & 1.0000 & 0.1765 \\
\hline$\tilde{\mathbf{X}}_{3}^{(1,3)}=\tilde{\mathbf{X}}_{3}^{(2,3)}$ & $\mathbf{X}_{3}=[11]$ & $\infty$ & - & - & 1.0000 & 1.0000 \\
\hline
\end{tabular}


Results in Table 2 indicate that $\Delta$ is greater than $\Delta_{0}$ for $\mathbf{X}_{1}$ and $\mathbf{X}_{3}$, but it is less than $\Delta_{0}$ for $\mathbf{X}_{0}$ and $\mathbf{X}_{2}$; thus, $\mathrm{P}\left(\Delta \geq \Delta_{0} \mid \mathbf{X}_{1}, d_{E}\right)=\mathrm{P}\left(\Delta \geq \Delta_{0} \mid \mathbf{X}_{3}, d_{E}\right)=1 \quad$ and $\mathrm{P}\left(\Delta \geq \Delta_{0} \mid \mathbf{X}_{0}, d_{E}\right)=\mathrm{P}\left(\Delta \geq \Delta_{0} \mid \mathbf{X}_{2}, d_{E}\right)=0$, which simplifies Eq. (13) to

$$
\mathrm{P}\left(g_{s}^{\text {serviceability }} \leq 0 \mid d_{E}\right)=\mathrm{P}\left(\mathbf{X}_{1} \mid d_{E}\right)+\mathrm{P}\left(\mathbf{X}_{3} \mid d_{E}\right)
$$

Hence, similar to the conditional probability of ultimate-strength failure in Eq. (16), the conditional probability of serviceability failure can be computed directly using the conditional probabilities of configurations.

We calculate the conditional probability of all the failure scenarios using the information in Table 2 and the proposed PRM. The computations based on Eqs. (7), (8), and (9) are illustrated in Figure 2. In this figure, based on Eq. (10), the adjustment factor for the first failure is

$$
\zeta_{i}^{(k, 1)}=\frac{1-(1-0.2994)(1-0.1710)}{(1-0.2994)(0.1710)+(0.2994)(1-0.1710)}=1.1394
$$

and the rest of the adjustment factors are 1 .

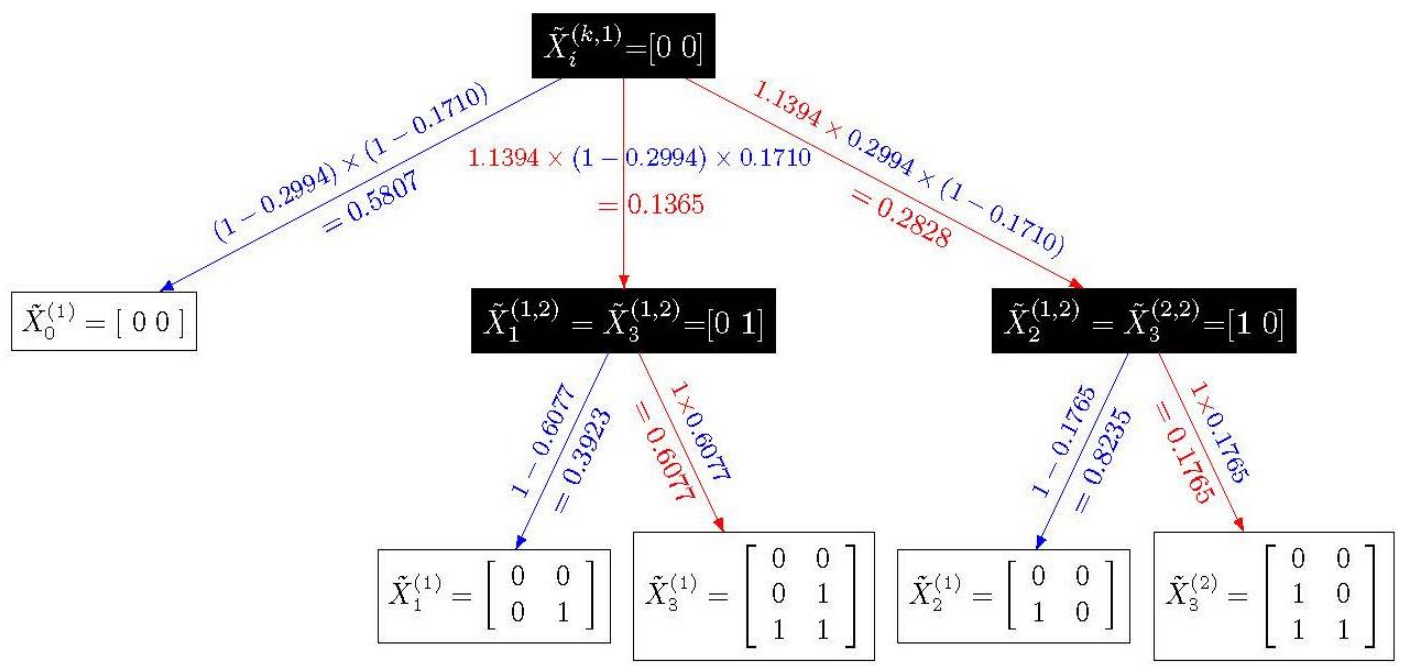

Figure 2- Calculating the conditional probabilities of configurations in the two-component structure

Using the results in Figure 2 and considering Eqs. (4) and (5), we compute the conditional probabilities of all the configurations as

$$
\begin{aligned}
& \mathrm{P}\left(\mathbf{X}_{0} \mid d_{E}\right)=\mathrm{P}\left(\tilde{\mathbf{X}}_{0}^{(1)} \mid d_{E}\right)=0.5807 \\
& \mathrm{P}\left(\mathbf{X}_{1} \mid d_{E}\right)=\mathrm{P}\left(\tilde{\mathbf{X}}_{1}^{(1)} \mid d_{E}\right)=0.3923 \times 0.1365=0.0535 \\
& \mathrm{P}\left(\mathbf{X}_{2} \mid d_{E}\right)=\mathrm{P}\left(\tilde{\mathbf{X}}_{2}^{(1)} \mid d_{E}\right)=0.8235 \times 0.2828=0.2329 \\
& \mathrm{P}\left(\mathbf{X}_{3} \mid d_{E}\right)=\mathrm{P}\left(\tilde{\mathbf{X}}_{3}^{(1)} \mid d_{E}\right)+\mathrm{P}\left(\tilde{\mathbf{X}}_{3}^{(2)} \mid d_{E}\right)=0.6077 \times 0.1365+0.1765 \times 0.2828=0.1329
\end{aligned}
$$


Please note that as it is required (configurations are mutually exclusive and collectively exhaustive),

$\mathrm{P}\left(\mathbf{X}_{0} \mid d_{E}\right)+\mathrm{P}\left(\mathbf{X}_{1} \mid d_{E}\right)+\mathrm{P}\left(\mathbf{X}_{2} \mid d_{E}\right)+\mathrm{P}\left(\mathbf{X}_{3} \mid d_{E}\right)=1$

These results can be used to calculate the conditional probabilities of failure of the system and its components. Using Eqs. (19) and (16), the conditional probabilities of serviceability and ultimate-strength failure given $d_{E}$ become 0.1864 and 0.1329 , respectively. Finally, using Eq. (18) and the probabilities in Table 2, the conditional probabilities of failure of the first and the second components under $d_{E}$ become 0.5721 and 0.3268 , respectively.

One of the applications of the proposed PRM is identifying the most critical failure scenarios or components of a system, which can be used for maintenance planning and failure prevention. By assessing the likelihood of each failure scenario or configuration and their influence on system functionality, the significance of each component to the system safety can be assessed and compared. For example, results in Table 2 show that an initial failure of Component 1 already corresponds to a system failure (because even if Component 2 does not fail, the system displacement still exceeds the allowable limit.) Conversely, the system could remain functional after the initial failure of Component 2. Therefore, Component 1 seems to be the more critical to the safety of this system. Using the calculations in Figure 2, initial failure of Component 1 has a (conditional) probability of about 0.1365 and thus the likelihood that the system failure occurs through an initial failure in Component 1 equals 0.1365 . In turn, if Component 2 initially fails, this system still has a 0.8235 chance of survival. Based on the calculated probabilities, the likelihood that this system loses its functionality through an initial failure of Component 2 is $0.2828 \times$ $0.1765=0.0499$. This implies that in the intact condition of this system, preventing the failure of Component 1 is about 2.7 times more important compared to Component 2 for maintaining the system safety. This information can be used as an input to maintenance planning of such system. Similar analysis can be performed in more complex systems to identify the likelihood of each failure scenarios and optimize their maintenance planning.

\section{Case Study: A Mooring System}

In this section, we show the application of the PRM for calculating the failure probability of offshore mooring systems. This section has two parts. First, the results of the PRM are compared with those we produced using Monte Carlo simulations following the algorithm developed by [9] for three mooring systems of different water depths under determined environmental loads. Then the PRM is used to identify a safer arrangement from among multiple mooring systems with a same number but different arrangements of the mooring lines under multidirectional environmental loads. Since the main function of a mooring system is to hold the vessel within allowable offset, a maximum allowable offset is used to define the system failure. However, because in practice it is common to set a limit for the allowable number of line failures, the effect of setting a limit for number of failed lines is also assessed.

The analyses for this study were performed using Mooring-3D [9] based on quasi-static method; however, the proposed method can be used based on more advanced analysis methods (e.g. dynamic time or frequency domain). For calculation of the mean capacity of the suction caisson, Mooring-3D used the method recommended by Aubeny et al. [15]. For probabilistic analysis of this study, Mooring-3D first calculated the internal forces in all the mooring components and the vessel offset under all configurations and environmental loads with $\mathrm{P}\left(g_{s}>0 \mid \mathbf{X}_{i}, d_{E}\right)=1$. Then, the PRM calculations or Monte Carlo simulations were performed. Because each mooring line is a series subsystem, one deterministic analysis 
was completed when assessing the component failures of each line under given demand and system configuration. In the Monte Carlo simulations, Mooring-3D was set to continue the simulations until either completing a hundred million realizations or reaching a C.O.V. of less than 0.005 for the system failure probability.

In the first part of this case study, results of the PRM are compared with those of Monte Carlo simulations for three typical catenary mooring systems located at 500, 1000, and $2000 \mathrm{~m}$ waters. All the three mooring systems held a spar with a radius of $25 \mathrm{~m}$. The allowable offsets of the spar under the determined 100 -year eastward loads of 850,1350 , and $800 \mathrm{kN}$ were 20,40 , and $60 \mathrm{~m}$ (4\% of the water depth) from its still position. The general properties of the mooring systems are given in Table 3. Figure 3 shows the intact configurations of the mooring lines in each system.
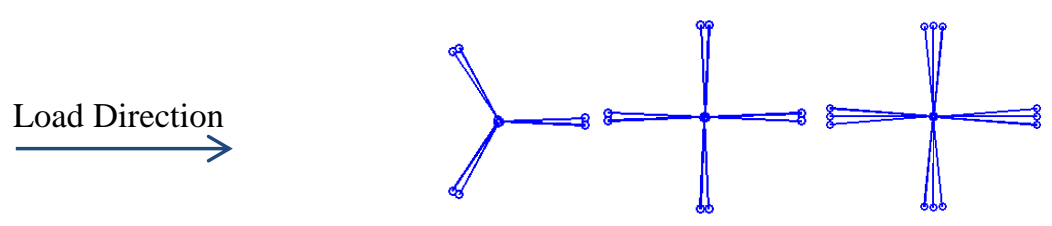

Figure 3- Schematic view of the mooring Systems; A (left), B (middle), and C (right)

Table 3. Basic Properties of the Mooring System in the First Part of the Case Study

\begin{tabular}{|c|c|c|c|c|c|c|c|}
\hline \multirow[t]{2}{*}{ ID } & \multirow[t]{2}{*}{ Arrangement } & \multirow[t]{2}{*}{$\begin{array}{l}\text { Water } \\
\text { Depth } \\
\text { (m) }\end{array}$} & \multirow{2}{*}{$\begin{array}{l}\text { Initial } \\
\text { Pretension } \\
\quad(\mathrm{KN})\end{array}$} & \multicolumn{3}{|c|}{$\begin{array}{l}\text { Mooring Line } \\
\text { Segment Type IDs } \\
\text { (Length, m) }\end{array}$} & \multirow[t]{2}{*}{$\begin{array}{l}\text { Suction Caisson } \\
\text { Diameter } \times \text { Length } \\
(\mathrm{m} \times \mathrm{m})\end{array}$} \\
\hline & & & & Top & Middle & Bottom & \\
\hline A & $3 \times 2$ & 500 & 850 & $\begin{array}{c}4 \\
(150)\end{array}$ & $\begin{array}{c}5 \\
(1200) \\
\end{array}$ & $\begin{array}{c}6 \\
(150)\end{array}$ & $1.5 \times 9.0$ \\
\hline B & $4 \times 2$ & 1000 & 1500 & $\begin{array}{c}4 \\
(200) \\
\end{array}$ & $\begin{array}{c}5 \\
(2000) \\
\end{array}$ & $\begin{array}{c}6 \\
(400) \\
\end{array}$ & $1.8 \times 10.8$ \\
\hline $\mathrm{C}$ & $4 \times 3$ & 1500 & 400 & $\begin{array}{c}3 \\
(200)\end{array}$ & $\begin{array}{c}2 \\
(2500)\end{array}$ & $\begin{array}{c}1 \\
(630)\end{array}$ & $1.1 \times 6.6$ \\
\hline
\end{tabular}

Each mooring line consisted of three segments and a suction caisson at the seafloor. Thus, the number of capacity random variables for Systems A, B, and C were 24, 32, and 48 respectively. In reference to Table 3 , the properties of the mooring segments types are provided in Table 4. All the mooring lines in each mooring system had the same properties. The seabed slope was taken to be negligible in all systems. Each mooring line was attached to a suction caisson at $67 \%$ of the length of the caisson below the seabed. The length-to-diameter ratios of all the suction caissons were taken to be six. The sizes of the suction caissons in each mooring system are also given in Table 3. The capacities of the suction caissons were calculated based on a soil shear strength of $3.8 \mathrm{kPa}(80 \mathrm{psf})$ at the seabed with a linear gradient of $1.57 \mathrm{kPa} / \mathrm{m}(10$ $\mathrm{psf} / \mathrm{ft}$ ) below the seabed; the coefficient of variations (C.O.V.) of the lateral capacity of the suction caissons was taken to be 0.3 [16]. Both the lateral capacity of the suction caissons and the tensile strength of the mooring line segments were assumed to follow lognormal distributions; however, a lower-bound capacity of 55\% the mean capacity was assumed for the suction caissons [16]. 
Table 4. Basic Properties of Mooring Line Segments

\begin{tabular}{ccccccc}
\hline Segment Type ID & 1 & 2 & 3 & 4 & 5 & 6 \\
\hline Type & $\begin{array}{c}\text { Wire } \\
\text { Rope }\end{array}$ & Polyester & Rig Wire & $\begin{array}{c}\text { R4 Anchor } \\
\text { Chain }\end{array}$ & $\begin{array}{c}\text { Neptune } \\
\text { Omega } \\
\text { Insert Wire }\end{array}$ & $\begin{array}{c}\text { R5 Rig } \\
\text { Chain }\end{array}$ \\
\hline $\begin{array}{c}\text { Unit Length Wet } \\
\text { Weight }\end{array}$ & $350 \mathrm{~N} / \mathrm{m}$ & $50 \mathrm{~N} / \mathrm{m}$ & $300 \mathrm{~N} / \mathrm{m}$ & $1264 \mathrm{~N} / \mathrm{m}$ & $334 \mathrm{~N} / \mathrm{m}$ & $1264 \mathrm{~N} / \mathrm{m}$ \\
\hline Friction Coefficient & 0.1 & 0.1 & 0.2 & 1 & 0.6 & 1 \\
\hline EA (stiffness) & $\begin{array}{c}4.7 \times 10^{5} \\
\mathrm{kN}\end{array}$ & $2.2 \times 10^{5} \mathrm{kN}$ & $\begin{array}{c}4.4 \times 10^{5} \\
\mathrm{kN}\end{array}$ & $6.0 \times 10^{5} \mathrm{kN}$ & $7.5 \times 10^{5} \mathrm{kN}$ & $\begin{array}{c}6.0 \times 10^{5} \\
\mathrm{kN}\end{array}$ \\
\hline $\begin{array}{c}\text { Mean Tensile } \\
\text { Strength }\end{array}$ & $7063 \mathrm{kN}$ & $7848 \mathrm{kN}$ & $6580 \mathrm{kN}$ & $6449 \mathrm{kN}$ & $8113 \mathrm{kN}$ & $8224 \mathrm{kN}$ \\
\hline Strength C.O.V. & $10 \%$ & $15 \%$ & $10 \%$ & $15 \%$ & $15 \%$ & $15 \%$ \\
\hline Resth
\end{tabular}

Results of probabilistic analysis of Systems A, B, and C using both the PRM and the Monte Carlo simulations are given in Table 5. The results are compared in two levels. First, the deterministic analysis was limited to the intact configuration only, thus, a large offset or any component failure defined a system failure. Then, the functionality of all the configurations with one failed line was also assessed, so the system failure was defined as if at least two components fail or the vessel offset exceed the allowable offset. Comparison between the results of the two methods in this table suggests that the differences between the results of these two methods are negligible. This indicates the significant advantage of the PRM because it is a closed-form solution and its computations are considerably faster than those of Monte Carlo simulations.

Table 5. Comparison between results of PRM and Monte Carlo simulations

\begin{tabular}{ccccc}
\hline \multirow{2}{*}{$\begin{array}{c}\text { Mooring } \\
\text { System ID }\end{array}$} & \multicolumn{4}{c}{ System Fragility } \\
\cline { 2 - 5 } & No Allowable Line Failure & One Allowable Line Failure \\
\cline { 2 - 5 } & PRM & Monte Carlo & PRM & Monte Carlo \\
\hline A & $1.92 \times 10^{-2}$ & $1.93 \times 10^{-2}$ & $1.91 \times 10^{-2}$ & $1.91 \times 10^{-2}$ \\
\hline B & $8.43 \times 10^{-3}$ & $8.52 \times 10^{-3}$ & $8.40 \times 10^{-3}$ & $8.47 \times 10^{-3}$ \\
\hline C & $8.05 \times 10^{-2}$ & $8.03 \times 10^{-2}$ & $8.00 \times 10^{-2}$ & $7.95 \times 10^{-2}$ \\
\hline
\end{tabular}

The second part of this case study compares the safety of some alternative arrangements of the 12 mooring lines of System C. As shown in Figure 4, the 12 mooring lines of System C can spread as individual lines (System D), or be arranged in three groups of four paired lines (System F), or in six groups of two paired lines (System H). Also, given the asymmetrical directional 100-year environmental loads as shown in Figure 5, rotating the asymmetrical Systems $\mathrm{C}$ and $\mathrm{F}$ for 30 degrees clockwise can lead to possibly safer Systems E and G respectively. Identifying the safest system from among all these 12-line alternatives is possible by calculating and comparing their fragilities using the PRM. In this assessment, again the system functionality is defined based on the vessel offset and the ultimate-strength failures of the lines. We considered three definitions for the system failure. The system was assumed to fail after either exceeding the allowable vessel offset of $60 \mathrm{~m}$ or the failure of at least one, two, or three lines. Therefore, the deterministic analyses were extended to all the possible system configurations with up to 2 failed lines. 
D)

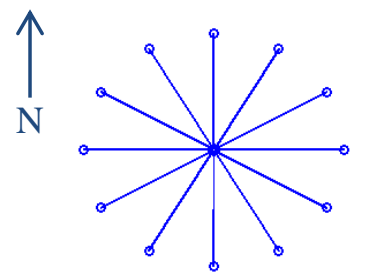

E)

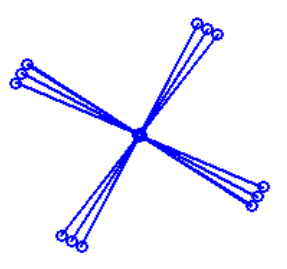

F)

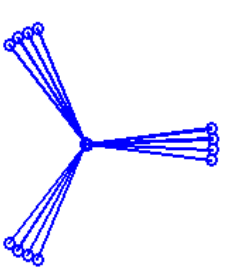

G)

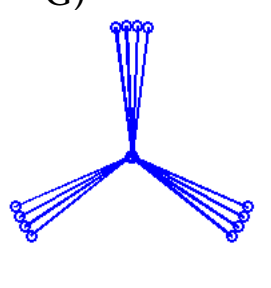

$\mathrm{H})$

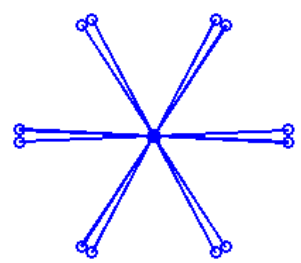

Figure 4. Schematic view of other alternative 12-line mooring systems

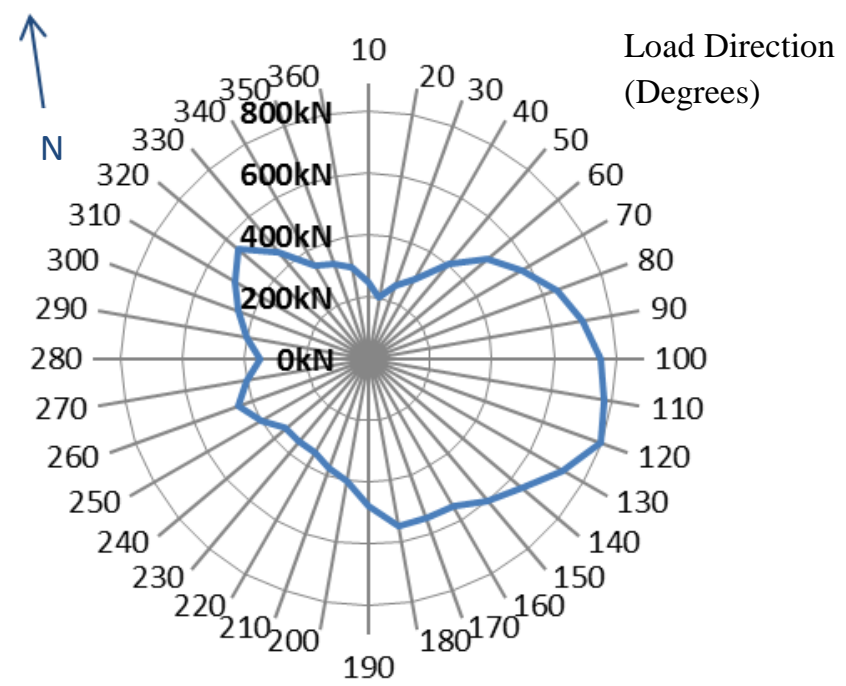

Figure 5. The solid line indicates the 100-year environmental load in each direction

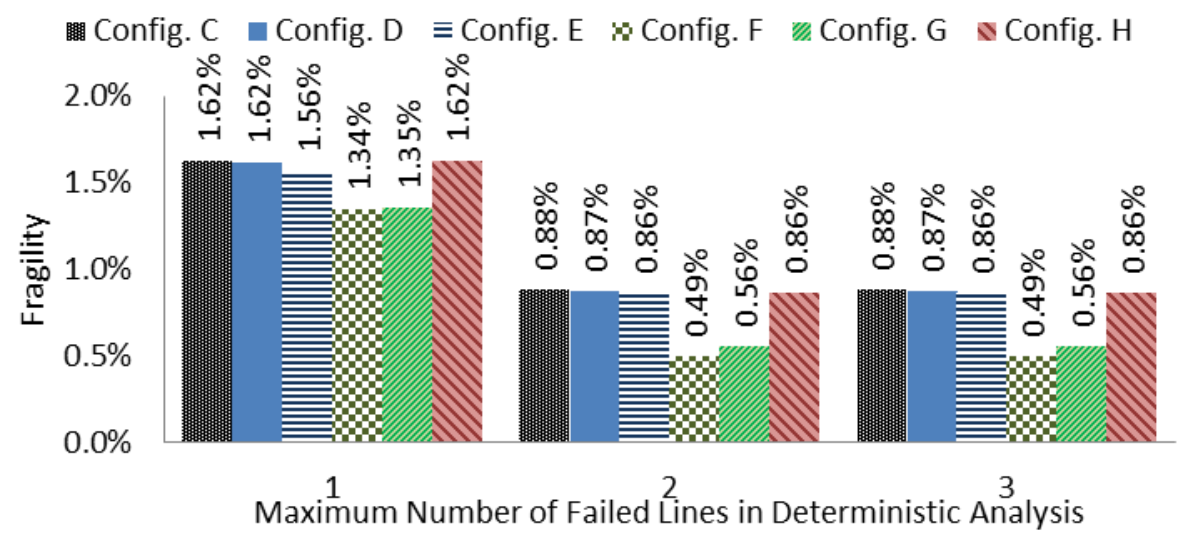

Figure 6. The fragilities of 12-line mooring systems

Fragility results of the PRM for the alternative systems with 12 mooring lines are shown in Figure 6. An overview of the results in this figure indicates that the calculated fragilities have reduced when a second line failure is also assessed compared to when a first line failure was considered only; however, 
accounting for a third line failure has not changed the fragilities. This indicates that after the two line failures, the system is no longer functional and thus the range of $d_{E}$ with $\mathrm{P}\left(g_{s}>0 \mid \mathbf{X}_{i}, d_{E}\right)=1$ is empty due to the vessel offset criterion; hence, this criterion governs such results. On the other hand, results in this figure show that the relative difference between the reliability of the alternative systems becomes more significant when accounting for higher number of progressive failures. For example, among the six analyzed arrangements of the 12-line mooring systems, System $\mathrm{F}$ has the highest and System $\mathrm{C}$ has the lowest safety. The fragility of System F is about $17 \%$ less than that of System $\mathrm{C}$ when the analysis accounts for the first failure only; however, this difference is increased to near $50 \%$ when the analysis is extended to a second line failure. These results highlight the significance of accounting for the damaged but functional configurations in the risk assessments. Results also suggest that Systems C, D, E, and H are equally safe despite the differences in their arrangement. In summary, System F is found to have the safest arrangement for 12-mooring lines for the given environmental load and alternative options.

\section{Conclusions}

This paper proposed a Progressive Reliability Method (PRM) for calculating the probability of failure of structural systems. The PRM is a closed-form solution based on known rules of probability to quantify the reliability of system functionality, ultimate-strength failure (exceeding a damage level), or component failures. In this method, all the possible system configurations in terms of the failure statuses of its components are first determined. By developing a fragility model, the response of the system under conditioned environmental loads is then evaluated. Using the probability density functions of the system component capacities (strengths) and results of structural (deterministic) analysis for the component demands (internal forces), the fragilities of the system under any configuration and environmental loads that is considered to be not-failed according to the definition of system failure is calculated. The total probability rule is then used to summarize the probabilistic results and calculate the system failure probability. The definition of failure in this method is flexible and can refer to the serviceability failure, the ultimate-strength failure of certain subsystems within the main system, a combination of the serviceability and ultimate-strength failures, or any other criteria as long as it can be checked given a configuration and demand. This method can be used to quantify the reliability and safety of complex structures. PRM can also be used to assess and compare the likelihoods of various failure scenarios and the importance of the system components to system safety, as illustrated in the example of a twocomponent structure. Such information can be also used as input for maintenance planning or risk assessments of the system components.

The PRM is illustrated in a case study for offshore mooring systems. First, the results of this method are compared with those of Monte Carlo simulations. Then a 12-line mooring system is analyzed under directional environmental loads to identify the safest arrangement and orientation among several possible options. The results show that the PRM results are very close to those of Monte Carlo simulations. They also indicated that for an example mooring system of this study, the reliability of this system is almost the same when the lines are arranged individually or in groups of two or three paired lines with different orientations. However, the reliability of the mooring system is considerably improved, up to $50 \%$, when the lines are formed in three groups of four and oriented against the peak directional loads. Results show that the PRM must be extended to two damaged lines to accurately quantify the reliability of system concerning the allowable vessel offset in this example. 


\section{References}

[1] Stiff J, Ferrari J, Ku A, Spong R. Comparative risk analysis of two fpso mooring configurations. Offshore Technology Conference 2013, Houston, TX, USA.

[2] Shah AA, Umar A, Siddiqui NA. A methodology for assessing the reliability of taut and slack mooring systems against instability. J Ocean Engineering 2005; 32: 1216-1234.

[3] Vazquez-Hernandez AO, Ellwanger GB, Sagrilo LVS. Reliability-based comparative study for mooring line design criteria. J Applied Ocean Research 2006; 28: 398-406.

[4] Montes-Iturrizaga R, Heredia-Zavoni E, Silva-Gonz'alez F. Short communication on the estimation of mooring line characteristic resistance for reliability analysis. J Applied Ocean Research 2007; 29: 239241.

[5] Grimea AJ, Langley RS. Lifetime reliability based design of an offshore vessel mooring. J Applied Ocean Research 2008; 30: 221-234.

[6] Ku A, Stiff J, Huang B, Puskar F. MODU mooring reliability analysis for hurricane condition in the gulf of mexico. Offshore Technology Conference 2009, Houston, TX, USA.

[7] Najjar SS, Gilbert RB. Importance of lower-bound capacities in the design of deep foundations. J Geotechnical and Geoenvironmental Engineering 2009; 135(7): 890-900.

[8] Montes-Iturrizaga R, Heredia-Zavoni E, Silvia-Gonzalez F, Straub D. Nested reliability analysis of mooring lines for floating systems. J Applied Ocean Research 2012; 34: 107-115.

[9] Mousavi ME, Gardoni P. Reliability-based design of offshore mooring systems. under review 2012

[10] Hernandez-Fajardo I. Probabilistic fragility of interdependent urban systems subjected to seismic hazards. Rice University 2012; PhD dissertation.

[11] Gardoni P, Der Kiureghian A, Mosalam KM. Probabilistic capacity models and fragility estimates for reinforced concrete columns based on experimental observations. J Engineering Mechanics 2012; 128(10): 1024-1038.

[12] Gardoni P, Mosalam KM, Der Kiureghian A. Probabilistic seismic demand models and fragility estimates for RC bridges. J Earthquake Engineering 2013; 7(sup001): 79-106.

[13] Ang AH, Tang WH. Probability concepts in engineering: emphasis on applications in civil \& environmental engineering. Wiley 2012

[14] Ethier SN, Kurtz TG. Markov processes: characterization and convergence. New York: John Wiley \& Sons Inc 1986.

[15] Aubeny CP, Murff JD, Moon SK. Lateral undrained resistance of suction caisson anchors. Int. J Offshore and Polar Engineering 2001; 11(3): 211-219. 
[16] Najjar SS, Gilbert RB. Importance of lower-bound capacities in the design of deep foundations. J Geotechnical and Geoenvironmental Engineering 2009; 135(7): 890-900. 